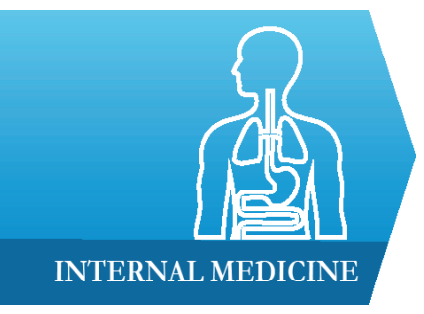

1) Cardiac Catheterization Laboratory, Department of Cardiology and Vascular Medicine, RSUD Pasar Rebo, East Jakarta, Indonesia

2) Faculty of Medicine, Universitas Muhammadiyah Prof. DR. Hamka, Tangerang, Indonesia

3) Faculty of Medicine, Universitas Diponegoro, Semarang, Indonesia
DOI: $10.15386 / \mathrm{mpr}-1884$

Manuscript received: 31.08 .2020

Received in revised form: 15.10 .2020

Accepted: 06.11.2020

Address for correspondence:

sidhilaksono@uhamka.ac.id

This work is licensed under a Creative Commons Attribution-NonCommercialNoDerivatives 4.0 International License

\title{
Metabolic syndrome and heart failure: mechanism and management
}

\author{
Sidhi Laksono Purwowiyoto ${ }^{1,2}$, Ananta Siddhi Prawara ${ }^{3}$
}

\begin{abstract}
Heart failure (HF) and metabolic syndrome (MetS) are syndromes that affect a large proportion of the world population. MetS is known to be one of the risk factors of $\mathrm{HF}$, and it can also act as comorbidity in HF. This review aims to further discuss the mechanism of MetS in causing HF, the management of MetS in order to prevent $\mathrm{HF}$, and the management of MetS in HF patients. Visceral adiposity is the primary trigger of MetS which is followed by chronic inflammation, insulin resistance, and neurohormonal activation. All the mechanisms causing MetS play also an important role in the progression of HF. The MetS approach can be achieved by managing its components according to the current guidelines and careful management of MetS should be done in patients with HF. MetS is closely related to the progression of HF so that comprehensive management which involves a multidisciplinary team is necessary for managing patients with metabolic syndrome and heart failure.
\end{abstract}

Keywords: chronic inflammation, insulin resistance, neurohormonal activation, visceral adiposity

\section{Introduction}

Heart failure (HF) is a clinical cardiac syndrome that affected more than 26 million of the world population [1]. The effort to prevent HF by controlling its risk factors before HF occurred need to be maximized because currently, no treatment can completely reverse or stop the progression of HF [2]. Metabolic syndrome (MetS) is a syndrome that affect, in our present days, many world populations due to sedentary lifestyle, overweight/obesity, and genetic factors [3]. The exact pathogenesis of MetS remained unidentified but it was suggested that the primary trigger to the syndrome was visceral adiposity, followed by insulin resistance, neurohormonal activation, and chronic inflammation [4]. MetS can be diagnosed with modified NCEP ATP III when fulfilling three or more criteria which are elevated waist circumference $(>102 \mathrm{~cm}$ for men and $>88 \mathrm{~cm}$ for women or $>90$ $\mathrm{cm}$ for Asian men and $\geq 80 \mathrm{~cm}$ for Asian women), elevated blood pressure $\geq 130$ / $\geq 85 \mathrm{mmHg}$, elevated fasting triglyceride (TG) $\geq 150 \mathrm{mg} / \mathrm{dl}$, reduced fasting highdensity lipoprotein (HDL) cholesterol level $(<40 \mathrm{mg} / \mathrm{dl}$ for men and $<50 \mathrm{mg} /$ dl for women), and elevated fasting blood sugar $\geq 100 \mathrm{mg} / \mathrm{dl}$ [3]. MetS and its component are involved as risk factors in HF development, while there is also a high prevalence of MetS components in patients with HF $[5,6]$. This review aimed to discuss the mechanism of metabolic syndrome in causing heart failure, the management of metabolic syndrome in order to prevent heart failure, and the management of metabolic syndrome in heart failure patients.

\section{Metabolic syndrome as a risk factor of heart failure}

Several studies have mentioned that MetS was the risk factor for heart failure. In a study conducted by Wilson et al., MetS increased the risk for cardiovascular disease (CVD) and type 2 diabetes mellitus (T2DM) in both men $(\mathrm{RR}=2.88$, 95\% CI 1.99 to 4.16$)$ and 
women ( $\mathrm{RR}=2.25,95 \% \mathrm{CI} 1.31$ to 3.88). Specifically, MetS was associated with the occurrence of more than one-third of CVD in men. However, in the study, CVD was defined broadly as the total events of stroke, intermittent claudication, and heart failure without mentioning the specific number of heart failure events [7]. The evidence of MetS being the risk factor of HF was further supported by a study in a large group of middle-aged men $(\mathrm{n}=2314)$ and a more specific outcome determining the relationship between MetS and HF. In multivariable Cox proportional hazard models adjusted for risk factors associated with HF including ECG finding of left ventricular hypertrophy, hypertension, diabetes, body mass index and smoking, the presence of MetS at the baseline was determined to be a significant predictor of HF (hazard ratio $1.66,95 \%$ CI 1.02 to 2.70 ) [8].

Tang et al. also confirmed the fact that MetS was an independent predictor of diastolic HF (DHF) and systolic HF (SHF) with $\mathrm{p}=0.049$ in a study with a total of 347 population. The study also found that beside MetS, hypertension was independently associated with DHF and SHF with $\mathrm{p}=0.028$ [9]. On the other hand, the study conducted by Hoetama et al. on a smaller population $(\mathrm{n}=228)$ found that metabolic syndrome itself was not counted as a risk factor for congestive HF (CHF), but its component which are raised blood pressure (OR 6.82, 95\% CI 1.47 to $31.53, \mathrm{p}=0.014$ ), while impaired glucose tolerance (OR $5.95,95 \%$ CI 3.26 to $10.85, \mathrm{p}=0.000$ ) showed a significant relationship with $\mathrm{CHF}[10]$.

\section{Mechanism of metabolic syndrome and its relation to heart failure \\ Visceral adiposity}

Adipose tissue is known to passively store excess energy in the form of triglyceride, and it is classified according to the location it can be found; subcutaneous and visceral [11]. Visceral adiposity was found to be associated with a higher risk of developing HF in the black population in the Jackson heart study [12]. The finding was supported with the outcome of MESA study, a large multiethnic U.S. study, which found that visceral adiposity was independently associated with the incidence of hospitalized patients with heart failure preserved ejection fraction (HFpEF) [13].

Visceral adiposity was also specifically stated to become the primary trigger of MetS and included in one of the diagnostic criteria of MetS because visceral adipose tissue encoded more adipocytokines, also known as adipokines, compared to subcutaneous adipose tissue $[11,14]$. Plasminogen activator inhibitor type 1 (PAI-1) that is known to be a strong risk factor for thrombotic diseases including myocardial infarction was expressed highly in adipose tissue [11]. Myocardial infarction (MI) was known to be able to develop to heart failure. The incidence of HF 7 years post-MI was recorded to be as high as $34.7 \%$ in 2596 patients [15].

Beside PAI-1, aminopeptidase M1 (apM-1) was another gene that was found abundantly in adipose tissue and its homozygous TT genotype was associated with a higher incidence of MetS. Furthermore, apM-1 gene encoded collagen-like protein that was called adiponectin [16]. Adiponectin was negatively correlated with BMI and the correlation between adiponectin level was stronger with visceral adiposity than subcutaneous adiposity. High adiponectin plasma concentration was associated with insulin sensitivity [11]. Adiponectin level was decreased as the result of the accumulation of macrophages in the adipose tissue that released proinflammatory cytokines during the chronic inflammatory state and it affected insulin sensitivity causing insulin resistance [17]. Insulin resistance was causing the impairment of glucose uptake, impaired suppression of hepatic glucose production, and lipolysis. Impaired glucose uptake and impaired suppression of hepatic glucose production resulting in elevated fasting blood sugar levels, while impairment of lipolysis caused more free fatty acid in the circulation [18]. The decreasing level of adiponectin itself is also correlated with increased blood pressure and triacylglycerol, and decreased HDL-cholesterol [19]. The low plasma concentration of adiponectin was also causing hypertension by impairing endotheliumdependent vasoreactivity [11]. Increasing renal sodium reabsorption was suggested to be the other mechanism that initiates hypertension in visceral adiposity. The increment of renal sodium reabsorption was caused by the activation of renin angiotensin aldosterone system (RAAS). RAAS was triggered by the compression of the kidney by visceral fat and the activation of the sympathetic nervous system (SNS) [20].

\section{Chronic inflammation}

Chronic inflammation process plays an important role in MetS. Mohammadi et al. found that serum IL-6 and TNF- $\alpha$ levels were significantly higher in patients with MetS [21]. The elevation of proinflammatory cytokine can also be detected in patients with heart failure. Elevated proinflammatory cytokines such as; tumor necrosis factor (TNF)- $\alpha$, interleukin (IL)-1, IL-6, galectin 3, soluble TNF receptor 1 and soluble TNF receptor 2 can be detected both in systolic heart failure and heart failure with preserved ejection fraction [22]. Furthermore, the elevated proinflammatory cytokine levels were also associated with the deterioration of functional class (New York Heart Association) and left ventricular ejection fraction [23].

TNF was able to provoke cardiomyocyte hypertrophy, activate neutral sphingomyelinase pathway that triggered apoptosis in isolated cardiomyocytes, and impair cardiac contractile function. TNF also induced the dilation of the left ventricle as a result of extracellular matrix degradation. The changes in the structure and function of the heart caused by TNF lead to the progression of heart failure [24]. IL-6 was involved in causing myocardial hypertrophy and left ventricular dysfunction [25]. Several studies have suggested the role of IL-6 in the progression of heart failure including 
its interaction with the vasculature and neurohormonal system to promote congestion, and its capability to reduce the phosphorylation of titin that caused the increased stiffness of cardiomyocyte [24,26].

\section{Insulin resistance}

Two pathways are responsible in the signaling pathway of insulin which are phosphatidylinositol 3 kinase (PI3-kinase)/protein kinase B (Akt) that mainly produce metabolic response and mitogen activated protein kinase (MAPK) that has growth factor-like response [27]. PI3kinase/Akt pathway activated ligand-activated insulin receptor that phosphorylated insulin receptor susbtrate-1 (IRS1). The phosphorylation of IRS1 activated downstream kinases that mediated several actions including the translocation of GLUT 4 to membrane. The translocation of GLUT 4 to membrane induces glucose uptake to the myocardium and skeletal muscle tissues, vasodilation with the help of nitric oxide, metabolic flexibility, and energy homeostasis [28]. On the other hand, MAPK pathway involving the tyrosine-phosphorylated IRS-1 in contributing to the growth and remodeling response. The response caused myocardial hypertrophy, cardiac fibrosis, impaired myocardial-endothelial signaling, and death of myocardial and endothelial cells [27]. Visceral adiposity altered the two pathways causing insulin resistance and insulin resistance impact the progression of HF through metabolic inflexibility, impaired calcium handling, mitochondrial dysfunction, oxidative stress, dysregulated myocardial-endothelial signaling causing energy deficiency, impaired diastolic dysfunction, myocardial cell death, and cardiac fibrosis [27]. A study conducted by Banerjee et al. showed that higher fasting insulin level was associated with the higher risk of HF incidence (HR 1.10, 95\% CI 1.05-1.15). The study also found that fasting insulin was positively correlated with adverse echocardiographic features such as left atrial size, left ventricular mass, and alterations in transmitral velocity [29].

\section{Neurohormonal activation}

The chronic activation of SNS and RAAS was one of the mechanisms that happened in MetS [20]. The chronic activation of the two neurohormonal systems caused a systemic effect that was harmful to the cardiovascular system. Two of the major systemic effect were the increase in blood pressure because of peripheral vasoconstriction and an increase in heart rate. The heart will compensate by increasing its contractility in order to meet cellular demand during the activation of neurohormonal systems [30]. The increase in contractility and heart rate lead to more energy demand, while in MetS, the energy produced was less than usual and the process was inefficient because of the switching from glucose metabolism to fatty acid oxidation (as a result of insulin resistance) [28]. Unable to compensate, cardiomyocyte hypertrophy occurrs and if the condition persists, remodeling may develop, and this process will progress to heart failure [31]. The relationship between visceral adiposity, chronic inflammation, insulin resistance, neurohormonal activation, metabolic syndrome, and heart failure is represented in figure 1 .

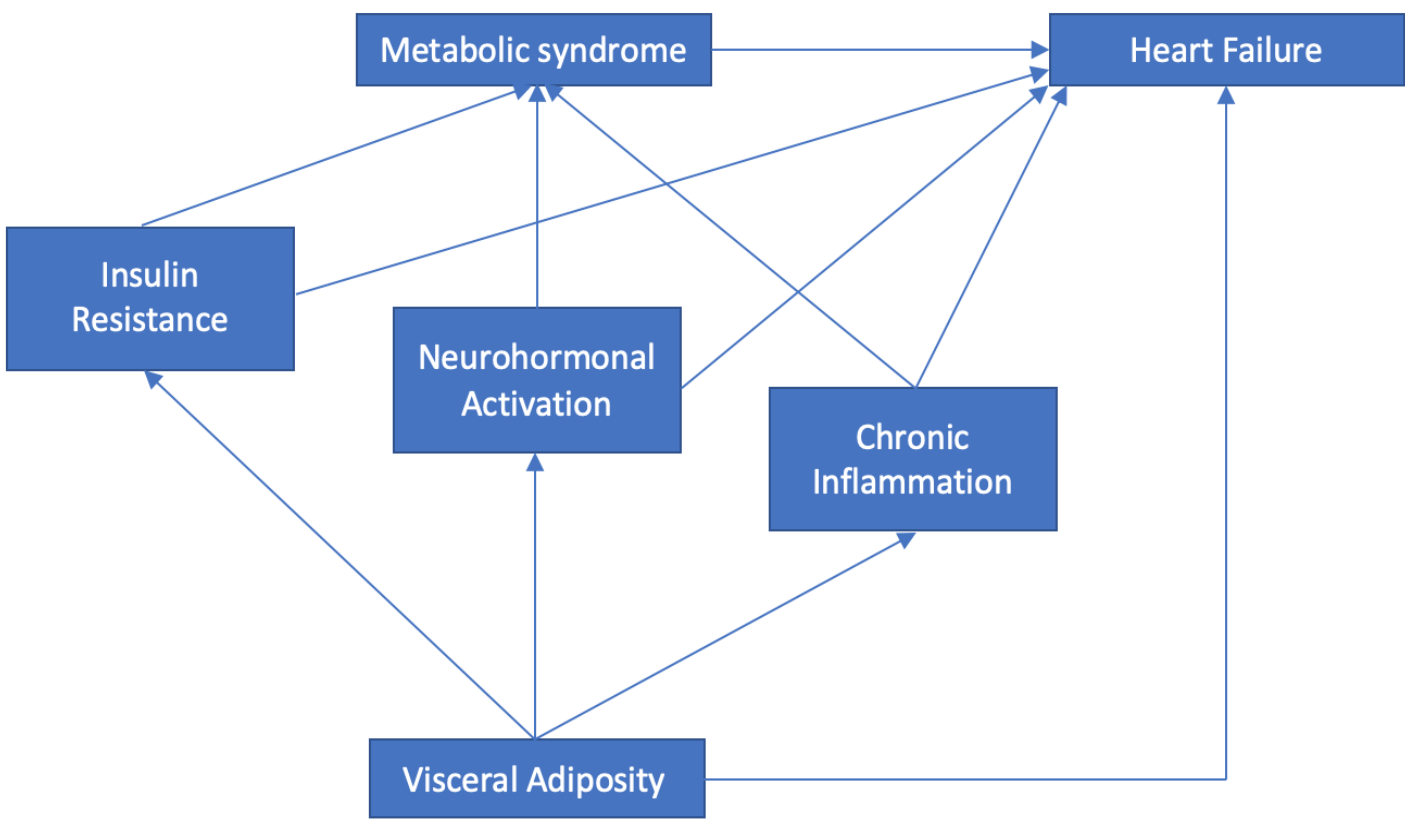

Figure 1. The relationship between visceral adiposity, chronic inflammation, neurohormonal activation, insulin resistance, metabolic syndrome, and heart failure. 


\section{Management of the metabolic syndrome in preventing heart failure}

Optimal metabolic syndrome management can be achieved with optimal lifestyle management and drug therapy. Diet, physical activity, and emotional regulation are part of lifestyle management, while drug therapy should focus on visceral adiposity, hypertension, hyperglycemia, and dyslipidemia [32]. As mentioned above, MetS was initiated by a sedentary lifestyle which caused visceral adiposity, the primary trigger of other MetS mechanisms $[3,4]$. Currently, waist circumference is used as the standard for measuring visceral adiposity in daily practice. The measurement of waist circumference should be done in a correct way to provide the most reliable data, namely at the midpoint between the lower border of the rib cage and the iliac crest. The cut-off points of waist circumference should be referred to the patient's race and gender [33,34].

Physical activity should be enhanced in patients with MetS. A meta-analysis by Ostman et al. proved that exercise training improved the outcomes in patients with metabolic syndrome. The patients included in the study varied in exercise intensity; moderate, vigorous, and high-intensity training. No statistically significant difference was found between the groups. However, isolated aerobic exercise was seen to be optimal in improving peak $\mathrm{VO}_{2}$, reducing fat mass, and body mass [35]. Another meta-analysis by Thorogood et al. concluded that moderate-intensity exercise programs for 6-12 months reduce the weight and waist circumference of the overweight and obese population modestly. Furthermore, isolated aerobic exercise improved systolic blood pressure, diastolic blood pressure, total cholesterol, and triglyceride levels modestly [36].

Long-term adherence to a healthy lifestyle is the key of lifestyle management for patients with metabolic syndrome. Several dietary patterns have been proved to ameliorate insulin resistance and MetS including Mediterranean-style, dietary approaches to stop hypertension (DASH), low-carbohydrate, and low-fat diets [37]. Neurohormonal activation is one of the mechanisms involved in MetS. Thus, emotional regulation, which impacted the neurohormonal mechanism, was related to MetS in both sexes and successful emotional regulation was leading to a reduced risk for MetS [38].

MetS management consensus or guideline is not available currently. Thus, the management of MetS should target every component of MetS that is presented in the patient. The hyperglycemia management of MetS patients can follow the consensus report by the American Diabetes Association (ADA) and the European Association for the Study of Diabetes (EASD): Management of Hyperglycemia in Type 2 Diabetes, released in 2018. The consensus report stresses the importance of lifestyle management, especially weight loss for diabetic patients with obesity, while also giving recommendations for medication management in patients with comorbidities such as clinical cardiovascular disease and chronic kidney disease [39]. The International Society of Hypertension released a new guideline for managing hypertension in 2020 from diagnosis to treatment. The guideline insured healthcare providers with two management options which are essential and optimal therapy [40]. In managing dyslipidemia, the 2019 European Society of Cardiology (ESC) and European Atherosclerosis Society (EAS) guidelines can be used as guidance. Strategies to control plasma cholesterol and triglycerides were provided in the guideline [41].

Monitoring for heart failure symptoms and signs was also important in managing MetS patients. The simplest form of monitoring can be done with history taking and physical examination. The occurrence of peripheral edema, breathlessness on exercise, or pulmonary rales are some signs highly suggestive for HF. Electrocardiogram can also be used to detect the anatomical abnormality of the heart such as left ventricle dilation [42]. The best modality that can be used in cardiac assessment is echocardiography being able to detect both anatomy and function of the heart including left ventricular ejection fraction parameter [43].

\section{Management of the metabolic syndrome in heart failure patients}

MetS was not included as an entity in the 2016 ESC guidelines for the diagnosis and treatment of acute and chronic heart failure but its components (diabetes, hypertension, and dyslipidemia) were mentioned as comorbidities [44]. Principally, the diabetes treatment in HF patients should be implemented gradually and moderately. The drug of choice recommended was metformin, which was also safe to be used in HFrEF patients $[39,44]$. The use of insulin in patients with HF were associated with negative outcomes. A large clinical trial done by Cosmi et al. assessed the effect of insulin on the outcomes of HF reduced ejection fractions (HFrEF) patients with diabetes mellitus (DM). The trials concluded that the use of insulin was associated with poor outcomes in HFrEF patients compared to patients that did not use insulin (OR 2.02, 95\% CI 1.87-2.19). Hypoglycemia, sodium, and water retention were the three factors suggested to be responsible for the outcome [45]. Thus, the risk and benefit of insulin use should be considered before prescribing to HF patients.

Hypertension management should be initiated with ACE-inhibitor or ARB. Angiotensin receptor neprilysin inhibitor (ARNI) can be considered as an alternative if the initial drugs failed. Beta-blocker, MRA, diuretic, hydralazine, or amlodipine can be administered and considered safe in systolic HF. Negatively inotropic CCBs are contraindicated for patients with HfrEF $[40,44]$. Reninangiotensin-aldosterone system inhibitors increase insulin sensitivity, while CCBs overdose increase insulin resistance [46]. Therefore, it will be beneficial to personalize the lowering blood pressure agent and monitor closely the dose of the drugs to avoid the risk of the patient getting 
extreme effects of hypoglycemia or hyperglycemia because of the drug mechanism. In managing dyslipidemia, the use of statin was not recommended in patients with HF, except for patients who are already receiving statins for CAD. There was no evidence for the use of PCSK9 inhibitors in HF patients. N-3 PUFAs can bring beneficial impact to HF patients when prescribed because it reduced $9 \%$ relative risk for mortality $[41,44]$.

\section{Obesity paradox in heart failure}

Obesity that was represented by visceral adiposity had been explained above as the primary trigger of metabolic syndrome. On the other hand, there were studies which proved that after heart failure developed, obesity in HF patients increased the patients' survival compared to normal weight patients. The study by Horwich et al. was the first study to conclude that obesity was not associated with increased mortality but rather with a more favorable prognosis [47]. Arena Ross et al. found that obesity improved the prognosis of patients with HF irrespective of its etiology, but HF etiology mattered when assessing the survival of HF patients [48]. In a prospective cohort held by Zamora et al., the obesity paradox was only found in patients with non-ischemic HF when using survival as the indicator [49]. One of the explanations to this paradox is that the more or less favorable clinical evolution of $\mathrm{HF}$ patients correlates with leptin/adiponectin ratio. Leptin, resistine, TNF-alpha factor exerts detrimental effects, while adiponectin seems to have a protective role [50]. On the other hand, leptin and adiponectin have a different effect in heart failure patients. Leptin was known to have an inverse correlation with mortality in heart failure patients, which suggested a protective role. Increased adiponectin levels in heart failure patients were associated with a severe prognosis, which may due to the regulatory mechanism to compensate the heart $[51,52]$. It was important to be noted that obesity paradox was etiology-dependent when the primary end-point was survival and the studies used body mass index (BMI) as an indicator of obesity, while, currently, waist circumference was used as the indicator of visceral adiposity in metabolic syndrome criteria. However, the obesity paradox in heart failures still needs to be taken into account when managing patients with obesity and heart failure.

\section{Conclusion}

Metabolic syndrome is closely related to the development of heart failure. Visceral adiposity acted as the primary trigger followed by chronic inflammation, insulin resistance, and neurohormonal activation. Each component of the metabolic syndrome affects the progression of heart failure. Prompt treatment of the metabolic syndrome will reduce the possibility of patients developing heart failure, and careful management of metabolic syndrome in heart failure patients will reduce the morbidity and mortality.
Comprehensive management involving a multidisciplinary team is necessary for managing patients with metabolic syndrome and heart failure. However, further research needs to be done to determine the best way to treat patients with metabolic syndrome and heart failure.

\section{References}

1. Savarese G, Lund LH. Global Public Health Burden of Heart Failure. Card Fail Rev. 2016;3:7-11.

2. Udelson JE, Stevenson LW. The Future of Heart Failure Diagnosis, Therapy, and Management. Circulation. 2016;133:2671-2686.

3. Grundy SM, Cleeman JI, Daniels SR, Donato KA, Eckel $\mathrm{RH}$, Franklin BA, et al. Diagnosis and management of the metabolic syndrome: an American Heart Association/ National Heart, Lung, and Blood Institute Scientific Statement. Circulation. 2005;112:2735-2752.

4. Rochlani Y, Pothineni NV, Kovelamudi S, Mehta JL. Metabolic syndrome: pathophysiology, management, and modulation by natural compounds. Ther Adv Cardiovasc Dis. 2017; 11:215-225.

5. Butler J. Primary prevention of heart failure. ISRN Cardiol. 2012;2012:982417.

6. Perrone-Filardi P, Paolillo S, Costanzo P, Savarese G, Trimarco B, Bonow RO. The role of metabolic syndrome in heart failure. Eur Heart J. 2015;36:2630-2634.

7. Wilson PW, D'Agostino RB, Parise H, Sullivan L, Meigs JB. Metabolic syndrome as a precursor of cardiovascular disease and type 2 diabetes mellitus. Circulation. 2005;112:30663072 .

8. Ingelsson E, Arnlöv J, Lind L, Sundström J. Metabolic syndrome and risk for heart failure in middle-aged men. Heart. 2006;92:1409-1413.

9. Tang ZH, Wang L, Zeng F, Zhang K. Association and predictive value analysis for metabolic syndrome on systolic and diastolic heart failure in high-risk patients. BMC Cardiovasc Disord. 2014;14:124.

10. Hoetama E, Hermawan B. Metabolic Syndrome: an Emerging Risk Factor for Congestive Heart Failure. Indonesian Journal of Cardiology. 2016;36:145-150.

11. Matsuzawa Y, Funahashi T, Nakamura T. The concept of metabolic syndrome: contribution of visceral fat accumulation and its molecular mechanism. J Atheroscler Thromb. 2011;18:629-639.

12. Pandey A, Kondamudi N, Patel KV, Ayers C, Simek S, Hall $\mathrm{ME}$, et al. Association Between Regional Adipose Tissue Distribution and Risk of Heart Failure Among Blacks. Circ Heart Fail. 2018;11:e005629.

13. Rao VS, Zhao D, Allison MA, Guallar E, Sharma K, Criqui $\mathrm{MH}$, et al. Adiposity and Incident Heart Failure and Its Subtypes: MESA (Multi-Ethnic Study of Atherosclerosis). JACC Heart Fail. 2018;6:999-1007.

14. Shah RV, Murthy VL, Abbasi SA, Blankstein R, Kwong $\mathrm{RY}$, Goldfine $\mathrm{AB}$, et al. Visceral adiposity and the risk of metabolic syndrome across body mass index: the MESA study. JACC Cardiovasc Imaging. 2014;7:1221-1235. 
15. Gerber Y, Weston SA, Enriquez-Sarano M, et al. Mortality Associated with Heart Failure after Myocardial Infarction: A Contemporary Community Perspective. Circ Heart Fail. 2016;9(1):1-9.

16. Barliana MI, Yolanda PD, Rostinawati $\mathrm{T}, \mathrm{Ng} \mathrm{H}$, Alfian $\mathrm{SD}$, Abdulah R, et al. Polymorphism of the APM1 gene in subjects with central obesity related to lower high-density lipoprotein cholesterol. Diabetes Metab Syndr Obes. 2019;12:2317-2324.

17. Kang YE, Kim JM, Joung KH, Lee JH, You BR, Choi MJ, et al. The Roles of Adipokines, Proinflammatory Cytokines, and Adipose Tissue Macrophages in Obesity-Associated Insulin Resistance in Modest Obesity and Early Metabolic Dysfunction. PLoS One. 2016;11:e0154003.

18. Riehle C, Abel ED. Insulin Signaling and Heart Failure. Circ Res. 2016;118:1151-1169.

19. Okamoto Y, Kihara S, Funahashi T, Matsuzawa Y, Libby P. Adiponectin: a key adipocytokine in metabolic syndrome. Clin Sci (Lond). 2006;110:267-278.

20. Hall JE, do Carmo JM, da Silva AA, Wang Z, Hall ME. Obesity-induced hypertension: interaction of neurohumoral and renal mechanisms. Circ Res. 2015;116:991-1006.

21. Mohammadi M, Gozashti MH, Aghadavood M, Mehdizadeh MR, Hayatbakhsh MM. Clinical Significance of Serum IL-6 and TNF- $\alpha$ Levels in Patients with Metabolic Syndrome. Rep Biochem Mol Biol. 2017;6:74-79.

22. Dick SA, Epelman S. Chronic Heart Failure and Inflammation: What Do We Really Know? Circ Res. 2016;119:159-176.

23. Gullestad L, Ueland T, Vinge LE, Finsen A, Yndestad A, Aukrust P. Inflammatory cytokines in heart failure: mediators and markers. Cardiology. 2012;122:23-35.

24. Murray DR, Freeman GL. Proinflammatory cytokines: predictors of a failing heart? Circulation. 2003;107:14601462.

25. Dutka M, Bobiński R, Ulman-Włodarz I, Hajduga M, Bujok J, Pająk C, et al. Various aspects of inflammation in heart failure. Heart Fail Rev. 2020;25:537-548.

26. Van Linthout S, Tschöpe C. Inflammation - Cause or Consequence of Heart Failure or Both? Curr Heart Fail Rep. 2017;14:251-265.

27. Aroor AR, Mandavia CH, Sowers JR. Insulin resistance and heart failure. Hear Fail Clin. 2012;8:609-617.

28. Gargiulo P, Marsico F, Renga F, Dell'Aversana S, Esposito I, Marciano C, et al. The metabolic syndrome in heart failure: insights to specific mechanisms. Heart Fail Rev. 2020;25(1):1-7.

29. Banerjee D, Biggs ML, Mercer L, Mukamal K, Kaplan $\mathrm{R}$, Barzilay $\mathrm{J}$, et al. Insulin resistance and risk of incident heart failure Cardiovascular Health Study. Circ Hear Fail. 2013;6:364-370.

30. Hartupee J, Mann DL. Neurohormonal activation in heart failure with reduced ejection fraction. Nat Rev Cardiol. 2017;14:30-38.

31. Tomek J, Bub G. Hypertension-induced remodelling: on the interactions of cardiac risk factors. J Physiol. 2017;595:40274036.

32. Wollina U. Recent advances in managing and understanding seborrheic keratosis. F1000Res. 2019;8:F1000 Faculty Rev1520 .

33. Camhi SM, Bray GA, Bouchard C, Greenway FL, Johnson WD, Newton RL, et al. The relationship of waist circumference and BMI to visceral, subcutaneous, and total body fat: sex and race differences. Obesity (Silver Spring). 2011;19:402-408.

34. Razi SM, Manish G, Keshav GK, Sukriti K, Gupta A. Site or Size of Waist Circumference, Which one is More important in Metabolic Syndrome? Int J Med Public Heal. 2016;6(2):69-72.

35. Ostman C, Smart NA, Morcos D, Duller A, Ridley W, Jewiss $D$. The effect of exercise training on clinical outcomes in patients with the metabolic syndrome: a systematic review and meta-analysis. Cardiovasc Diabetol. 2017;16:110.

36. Thorogood A, Mottillo S, Shimony A, Filion KB, Joseph $\mathrm{L}$, Genest $\mathrm{J}$, et al. Isolated aerobic exercise and weight loss: a systematic review and meta-analysis of randomized controlled trials. Am J Med. 2011;124:747-755.

37. Hoyas I, Leon-Sanz M. Nutritional Challenges in Metabolic Syndrome. J Clin Med. 2019;8:1301.

38. Kinnunen ML, Kokkonen M, Kaprio J, Pulkkinen L. The associations of emotion regulation and dysregulation with the metabolic syndrome factor. J Psychosom Res. 2005;58:513521.

39. Davies MJ, D'Alessio DA, Fradkin J, Kernan WN, Mathieu C, Mingrone G, et al. Management of Hyperglycemia in Type 2 Diabetes, 2018. A Consensus Report by the American Diabetes Association (ADA) and the European Association for the Study of Diabetes (EASD). Diabetes Care. 2018;41:2669-2701.

40. Unger T, Borghi C, Charchar F, Khan NA, Poulter NR, Prabhakaran D, et al. 2020 International Society of Hypertension Global Hypertension Practice Guidelines. Hypertension. 2020:75:1334-1357.

41. Mach F, Baigent C, Catapano AL, Koskinas KC, Casula M, Badimon L, et al. 2019 ESC/EAS Guidelines for the management of dyslipidaemias: lipid modification to reduce cardiovascular risk. Eur Heart J. 2020;41:111-188.

42. Pradedova EV, Isheeva OD, Salyaev RK. Classification of the antioxidant defense system as the ground for reasonable organization of experimental studies of the oxidative stress in plants. Russ J Plant Physiol. 2011;58:210-217.

43. Modin D, Andersen DM, Biering-Sørensen T. Echo and heart failure: when do people need an echo, and when do they need natriuretic peptides? Echo Res Pract. 2018;5:R65-R79.

44. Ponikowski P, Voors AA, Anker SD, Bueno H, Cleland JGF, Coats AJS, et al. 2016 ESC Guidelines for the diagnosis and treatment of acute and chronic heart failure: The Task Force for the diagnosis and treatment of acute and chronic heart failure of the European Society of Cardiology (ESC) Developed with the special contribution of the Heart Failure Association (HFA) of the ESC. Eur Heart J. 2016;37:21292200 .

45. Cosmi F, Shen L, Magnoli M, Abraham WT, Anand IS, Cleland JG, et al. Treatment with insulin is associated with worse outcome in patients with chronic heart failure and diabetes. Eur J Heart Fail. 2018;20:888-895. 
46. Rizos CV, Elisaf MS. Antihypertensive drugs and glucose metabolism. World J Cardiol. 2014;6:517-530.

47. Horwich TB, Fonarow GC, Hamilton MA, MacLellan WR, Woo MA, Tillisch JH. The relationship between obesity and mortality in patients with heart failure. J Am Coll Cardiol. 2001;38:789-795.

48. Arena R, Myers J, Abella J, Pinkstaff S, Brubaker P, Moore $\mathrm{B}$, et al. Influence of etiology of heart failure on the obesity paradox. Am J Cardiol. 2009;104:1116-1121.

49. Zamora E, Lupón J, de Antonio M, Urrutia A, Coll R, Díez $\mathrm{C}$, et al. The obesity paradox in heart failure: is etiology a key factor? Int J Cardiol. 2013;166:601-605.

50. Wannamethee SG, Shaper AG, Whincup PH, Lennon L, Papacosta O, Sattar N. The obesity paradox in men with coronary heart disease and heart failure: the role of muscle mass and leptin. Int J Cardiol. 2014;171:49-55.

51. Goldstein BJ, Scalia RG, Ma XL. Protective vascular and myocardial effects of adiponectin. Nat Clin Pract Cardiovasc Med. 2009;6:27-35.

52. Van de Voorde J, Pauwels B, Boydens C, Decaluwé K. Adipocytokines in relation to cardiovascular disease. Metabolism. 2013;62:1513-1521. 